\title{
Implicit Gaze-Assisted Adaptive Motion Scaling for Highly Articulated Instrument Manipulation
}

\author{
Gauthier Gras $^{1}$, Konrad Leibrandt ${ }^{1}$, Piyamate Wisanuvej ${ }^{1,2}$, Petros Giataganas ${ }^{1}$, Carlo A. Seneci ${ }^{1}$, \\ Menglong $\mathrm{Ye}^{1}$, Jianzhong Shang ${ }^{1}$, Guang-Zhong Yang ${ }^{1}$, Fellow, IEEE
}

\begin{abstract}
Traditional robotic surgical systems rely entirely on robotic arms to triangulate articulated instruments inside the human anatomy. This configuration can be ill-suited for working in tight spaces or during single access approaches, where little to no triangulation between the instrument shafts is possible. The control of these instruments is further obstructed by ergonomic issues: The presence of motion scaling imposes the use of clutching mechanics to avoid the workspace limitations of master devices, and forces the user to choose between slow, precise movements, or fast, less accurate ones. This paper presents a bi-manual system using novel self-triangulating 6-degrees-of-freedom (DoF) tools through a flexible elbow, which are mounted on robotic arms. The control scheme for the resulting 9-DoF system is detailed, with particular emphasis placed on retaining maximum dexterity close to joint limits. Furthermore, this paper introduces the concept of gaze-assisted adaptive motion scaling. By combining eye tracking with hand motion and instrument information, the system is capable of inferring the user's destination and modifying the motion scaling accordingly. This safe, novel approach allows the user to quickly reach distant locations while retaining full precision for delicate manoeuvres. The performance and usability of this adaptive motion scaling is evaluated in a user study, showing a clear improvement in task completion speed and in the reduction of the need for clutching.
\end{abstract}

\section{INTRODUCTION}

Recent advances in surgical robots have provided surgeons with enhanced dexterity, consistency and accuracy for a range of minimally invasive procedures [1]. The development of complex wristed instruments for minimally invasive surgery (MIS) has greatly contributed to the success of these systems. However, traditional robotic systems such as the da Vinci ${ }^{\circledR}$ (Intuitive Surgical Inc., CA, United States) typically rely on supporting robotic arms for the triangulation of these instruments. This is effective for certain categories of procedures such as in general abdominal surgery, where a large workspace is available and the instrument shafts can come through distant entry ports. However, this approach can present significant drawbacks when operating in tight spaces or along narrow anatomical pathways [2], [3]. In cases such as Transanal Endoscopic Micro-Surgery (TEMS), Ear, Nose and Throat (ENT) surgery, and keyhole neurosurgical approaches the instrument shafts are constrained to be nearly parallel, and as such the supporting robotic arms cannot offer the triangulation necessary [4].

This work is supported by the Department of Health and Wellcome Trust through the Health Innovation Challenge Fund (HICF-T4-299).

${ }^{1}$ All authors are with the Hamlyn Centre for Robotic Surgery, Imperial College London, London, United Kingdom.

${ }^{2} \mathrm{P}$. Wisanuvej is also with Kasetsart University, Bangkok, Thailand. Corresponding author: gauthier.gras12@imperial.ac.uk

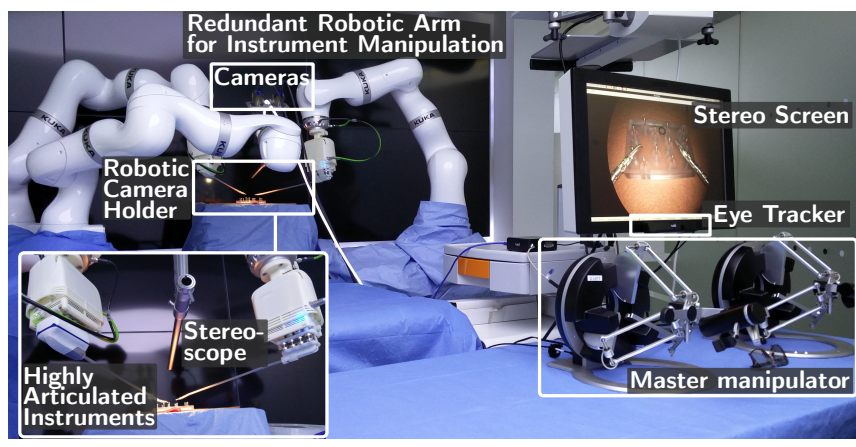

Fig. 1. Overview of the proposed adaptive motion scaling system.

In order to mitigate the need for the robotic arms to provide the triangulation, highly articulated instruments with an inherent capability to bend can be developed. This is achieved by increasing the number of degrees-of-freedom (DoF) available to the instruments, effectively increasing their dexterous workspace. However, to keep transmitted forces high, the joint segments should remain short, leading to joints often operating close to or at their joint limits. The control of these instruments thus needs to take particular care of the kinematic challenges when operating near the joint limits [5], [6]. Previous work on highly dexterous instruments has studied the control challenges of such devices in single access surgery [7], [8], [9]. This work attempts to merge the advantages of these approaches with those associated with the use of external robotic arms. A control scheme for novel 6-DoF instruments possessing a flexible elbow mounted on robotic arms is presented. The inverse kinematic approach used to control a combined total of 9-DoF per robot instrument is detailed, placing particular emphasis on maintaining dexterity close to the joint limits.

In addition to the mechanical and kinematic limitations outlined above, existing ergonomic issues also limit several of the main advantages of robotic systems. In systems such as the da Vinci the surgeon is able to control the camera directly but is required to clutch the instruments before being able to do so, thus preventing simultaneous control of the instruments and the camera. Similarly, while motion scaling allows the surgeon to perform fine and precise movements it also prevents the surgeon from making larger movements without having to clutch and reposition the master device end-effectors. This problem is all the more noticeable when using master devices with a smaller workspace. Combined, 
these issues can make navigating around the operating site time consuming and frustrating by requiring the user to clutch the instruments and the camera multiple times.

Past work has shown the potential of using eye-tracking to alleviate these issues [10], [11]. The presented work builds on the authors' previous experience in intention recognition for camera control [12] to introduce an intuitive way of providing adaptive motion scaling. In this paper, eye-tracking data is combined with hand motion information obtained from the master device, as well as tool tracking information to infer where the user is trying to move the instruments. The scaling factor of the master device is modulated accordingly, allowing the user to quickly reach distant locations while retaining precise control for delicate manipulations. Unlike in [13] where an eye-tracking approach is used to provide haptic guidance towards a visual point of interest, no external forces or motions are generated using this approach. A user study is conducted to evaluate the effectiveness of this approach, showing a clear reduction in task execution times and number of times clutched.

\section{System Overview AND Design}

The presented system uses three KUKA LBR iiwa 7-DoF redundant robotic arms (KUKA Roboter GmbH, Germany) for the global positioning of two flexible instruments and a stereoscope. The 6-DoF flexible instruments are attached onto the robotic arms via custom-designed actuation units. These actuation units house the hardware components required for the motion of the tools. This comprises Maxon EC brushless DC motors (Maxon Motor, Switzerland), custom designed motor controller modules and an interface motherboard. The power line and communication bus for each of the flexible instruments are routed internally through the corresponding robotic arm and connected via a short cable from the arm's flange, to prevent cable tangling during large movements. The user interacts with the system via two Omega.7 haptic devices (Force Dimension, Switzerland), and a Tobii Pro X2-60 eye tracker (Tobii AB, Sweden). The system setup is shown in Fig. 1.

The 6-DoF instruments are fabricated with stainless steel using an additive manufacturing process (Selective Laser Melting - SLM). For a detailed analysis on SLM manufacturing of surgical instruments, please refer to [14]. Each

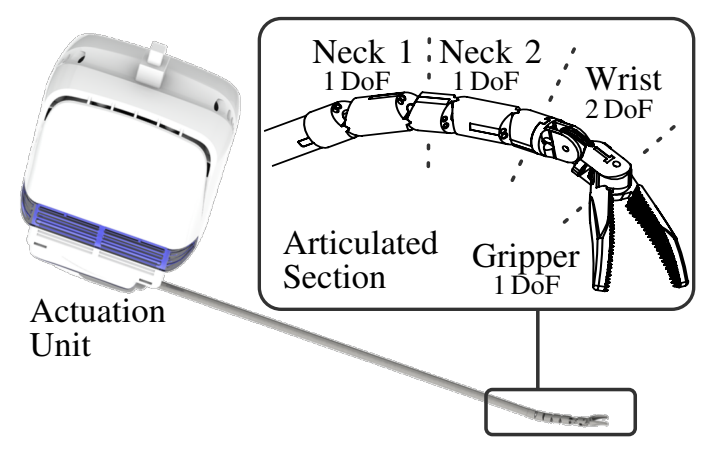

Fig. 2. Actuation unit and articulated instrument section.

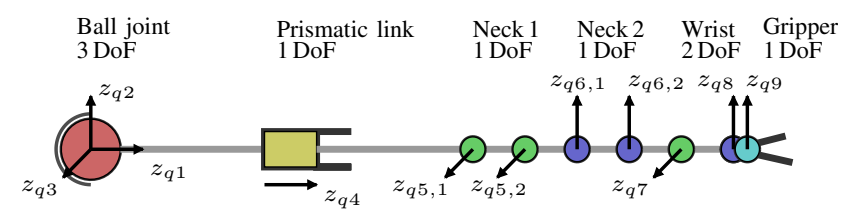

Fig. 3. Illustration of the kinematic chain of the virtual 9 DoF robotic manipulator. Featuring dependent joints in neck 1 and neck 2, and a wrist with $2 \mathrm{DoF}$, where the pitch is actuated by $q_{7}$, the yaw is the mean of $q_{8}$ and $q_{9}$, and the gripper DoF is represented by the angle difference of $q_{8}$ and $q_{9}$.

instrument has an overall diameter of $5.5 \mathrm{~mm}$, complete shaft length of $250 \mathrm{~mm}$ and flexible tip length of $50 \mathrm{~mm}$, enabling it to explore tight operating sites effectively. The transmission of the actuation is based on tendons that are affixed to capstan mechanisms through a pulley system. The capstan mechanisms are coupled with the motors in the proximal actuation system. The latter is enclosed in a custom designed casing that is rigidly attached to the robotic arm. The casing is fabricated with sterilisable, thermoplastic ABS material using a rapid prototyping process (Fused Deposition Modelling - FDM). It features an efficient airflow design that reduces the temperature in the motor chamber from $80^{\circ} \mathrm{C}$ (without fan) to $35^{\circ} \mathrm{C}$. The efficiency of the cooling design is particularly important due to the miniaturized actuation pack design which houses motors and control electronics, and which produces a lot of heat energy. Fig. 2 shows the surgical instrument and actuation pack.

\section{INSTRUMENT AND SyStem CONTROL}

\section{A. Instrument Kinematics}

Both 9-DoF tools are controlled using a combined kinematics scheme between the redundant robotic arms and the flexible instruments. This is achieved by considering a virtual 9-DoF manipulator, see Fig. 3. One DoF $\left(n_{g}=1\right)$ represents the opening angle of the gripper, and the remaining $8 \mathrm{DoF}$ $\left(n_{k}=8\right)$ are used for positioning and orienting the tool endeffector in the surgical task space. The base of the virtual tool is placed at the surgical port, where the tool enters the workspace, and where a virtual ball joint provides pivoting motion. The axial rotation of the virtual tool as well as the five distal DoFs are actuated by the 6-DoF instrument and are directly mapped to the joint-space of the flexible instrument. The remaining $3 \mathrm{DoF}$ of the virtual tool are actuated by the

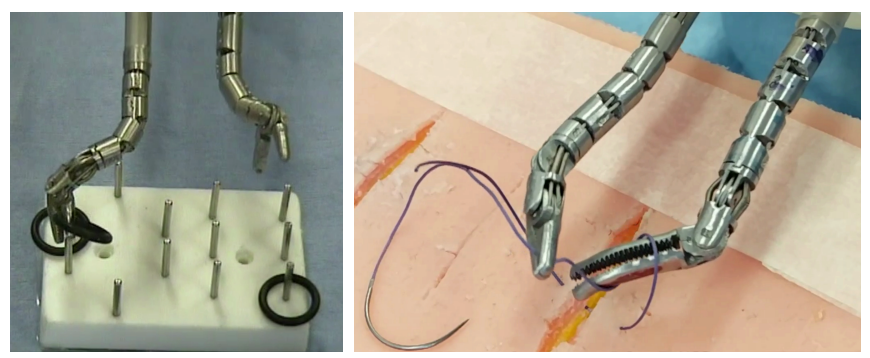

Fig. 4. Robotic instruments in parallel configuration as used in single port access surgery. 
redundant robotic arms, where the pitch and yaw rotation angles of the ball joint and the translation of the prismatic link are used to construct the corresponding end-effector poses of the robotic arms.

The manufacturer-provided inverse kinematic solver is used to control the robotic arms. The inverse kinematic problem of the virtual tool is solved using the Jacobian $\left(\boldsymbol{J} \in \mathbb{R}^{6 \times n_{k}}, n_{k}=8\right)$ based Newton-Raphson method. The developed inverse kinematic scheme is designed to perform well in conventional laparoscopic procedures, and to also be suitable for surgical interventions permitting only very limited triangulation at the port. This last configuration results in close-to-parallel shafts, significantly limiting the operation space of the ball joint in its pitch and yaw motion, as illustrated in Fig. 4. In such configurations the positioning of the end-effector has to be provided mainly by the flexible instruments. Due to the relatively short length of the articulated instrument tips, manipulation close to the joint limits is likely in such situations.

Based on these considerations as well as surgeon feedback, the task space $\left(\boldsymbol{x}=\left[t_{x}, t_{y}, t_{z}, r_{x}, r_{y}, r_{z}\right]^{T}\right)$ is separated into primary $\left(\boldsymbol{x}_{p}=\left[t_{x}, t_{y}, t_{z}, r_{z}\right]^{T}\right)$ and secondary $\left(\boldsymbol{x}_{s}=\left[r_{x}, r_{y}\right]^{T}\right)$ task-spaces. The primary task-space represents the position and instrument roll whereas the secondary task-space represents the remaining orientational DoFs (pitch, yaw). Consequently, the primary Jacobian $\boldsymbol{J}_{p} \in$ $\mathbb{R}^{4 \times n_{k}}$ consists of the first three rows and the last row of $\boldsymbol{J}$; and $\boldsymbol{J}_{s} \in \mathbb{R}^{2 \times n_{k}}$ consist of the fourth and fifth row of $\boldsymbol{J}$. The secondary task goals are optimized using the null-space of the primary goals, for which the pseudo-inverse Jacobian $\boldsymbol{J}^{\star}$ is calculated based on the damped-least-square (DLS) method by

$$
\boldsymbol{J}^{\star}=\boldsymbol{V} \operatorname{diag}\left(\frac{1}{\tilde{\sigma}_{1}}, \ldots, \frac{1}{\tilde{\sigma}_{n}}\right) \boldsymbol{U}^{T},
$$

where $\boldsymbol{U}, \boldsymbol{V}$ are right and left singular vectors provided by a singular value decomposition (SVD) of $\boldsymbol{J} . \tilde{\sigma}_{i}^{2}$ is the modified $i^{\text {th }}$ eigenvalue potentially damped by,

$$
\tilde{\sigma}_{i}=\left\{\begin{array}{ll}
\sigma_{i} & , \text { if } \min _{j}\left(\left|\sigma_{j}\right|\right) \geq \sigma_{\min } \\
\left(\sigma_{i}{ }^{2}+\lambda_{t}{ }^{2}\right) \sigma_{i}{ }^{-1} & , \text { else }
\end{array},\right.
$$

and with

$$
\lambda_{t}^{2}=\lambda_{\max }^{2}\left[1-\left(\min _{j}\left(\left|\sigma_{j}\right|\right) \sigma_{\min }{ }^{-1}\right)^{2}\right],
$$

where $i, j \in\{1, \ldots n\}$. This provides stable manipulation close to singular configurations, by avoiding excessive jointvelocities. However, this comes at the cost of slower convergence rates in the optimization process, and a suboptimal null-space mapping, therefore the damping in (2) is only active in case the smallest singular value is below a critical singular value threshold. Furthermore, in (3) the damping is increased the smaller the minimal singular value is. The damped inverse Jacobian for the primary $\left(\boldsymbol{J}_{p} \in \mathbb{R}^{n_{k} \times 4}\right)$ and secondary $\left(\boldsymbol{J}_{s} \in \mathbb{R}^{n_{k} \times 2}\right)$ task-space are calculated with (1),

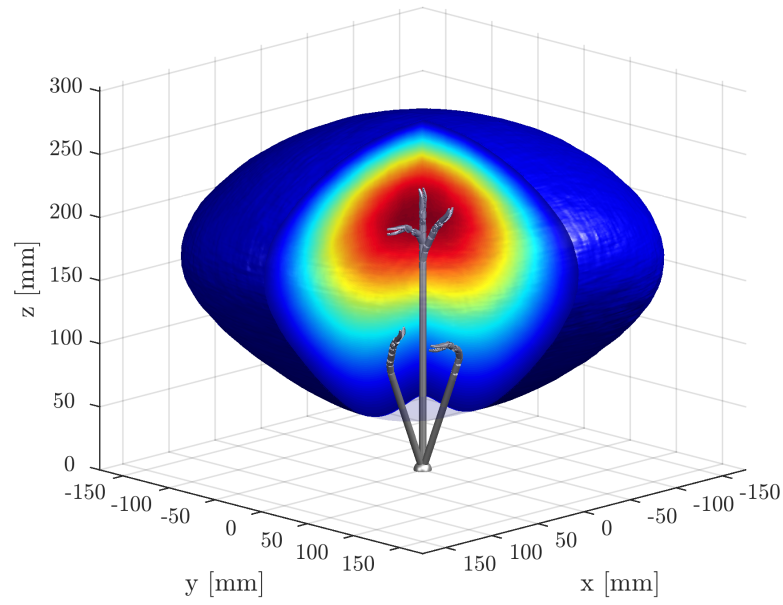

Fig. 5. Dexterity $\mathcal{D}$ analysis of the virtual instrument, using 1E9 random robot configurations. Red: high dexterity, blue: low dexterity.

and to reach the desired end-effector pose $\boldsymbol{T}_{e, d}$ the jointvalues of the virtual tool are iteratively updated with

$$
\begin{aligned}
\boldsymbol{q}_{t+1} & =\boldsymbol{q}_{t}+\Delta \boldsymbol{q}_{t+1}=\boldsymbol{q}_{t}+\dot{\boldsymbol{q}}_{t} \Delta t, \\
\dot{\boldsymbol{q}}_{t} & =\boldsymbol{J}_{p}^{\star} \dot{\boldsymbol{x}}_{p}+\left(\boldsymbol{I}_{4}-\boldsymbol{J}_{p}^{\star} \boldsymbol{J}_{p}\right) \dot{\boldsymbol{q}}_{\text {null }}, \\
\dot{\boldsymbol{q}}_{\text {null }} & =\boldsymbol{f}_{\text {ori }}\left(\dot{\boldsymbol{x}}_{p}, \dot{\boldsymbol{x}}_{s}, \boldsymbol{J}_{s}^{\star}\right)+\boldsymbol{f}_{\text {qlim }}\left(\boldsymbol{q}_{t}\right),
\end{aligned}
$$

where the null-space of the primary optimization goal is used in (5) to optimize for secondary goals in (6). The secondary goals are i. the yaw, pitch-orientation of the end-effector pose $\left(f_{\text {ori }}\right)$, and ii. joint-limit avoidance $\left(f_{\text {qlim }}\right)$. They are defined as,

$$
\begin{aligned}
\boldsymbol{f}_{\text {ori }} & =k_{\text {ori }}\left(1-\frac{\min \left(\left\|\dot{\boldsymbol{x}}_{p}\right\|, \dot{x}_{\mathrm{p}, \max }\right)}{\dot{x}_{\mathrm{p}, \max }}\right) \boldsymbol{J}_{\mathrm{s}}^{\star} \dot{\boldsymbol{x}}_{\mathrm{s}}, \\
f_{\mathrm{qlim}, i} & =k_{\mathrm{qlim}}\left|\frac{q_{i, \max }+q_{i, \min }-2 q_{i}}{q_{i, \max }-q_{i, \min }}\right|^{k_{e}} \\
& \cdot \operatorname{sgn}\left(q_{i}-\frac{q_{i, \max }+q_{i, \min }}{2}\right) .
\end{aligned}
$$

The weight in (7) is dependent on the primary task-space velocity $\dot{\boldsymbol{x}}_{p}$ to ensure that primary goals are met first even when the null-space mapping is suboptimal. Furthermore, the exponent $k_{e}>1$ (here $k_{e}: 3$ ) in (8) emphasises join-limit avoidance only for configurations close to the limits which allows to optimize mainly for the orientation goals. However, joint limit avoidance cannot guarantee that joint limits are in fact completely avoided, in particular if the desired pose is in locations of low dexterity or outside of the reachable workspace.

\section{B. Dexterity Analysis for Task Space Restriction}

While the previous section addressed the challenge of reaching joint limits in joint-space, a complementary method based on workspace analysis [15] is used to restrict the endeffector set point $\boldsymbol{T}_{e, d}$ in task space, as illustrated in Fig. 5. The dexterity measure $\mathcal{D}$ is calculated as,

$$
\mathcal{D}=\mathcal{L}_{q} \mathcal{M}=\mathcal{L}_{q} \sqrt{\left|\boldsymbol{J} \boldsymbol{J}^{T}\right|},
$$




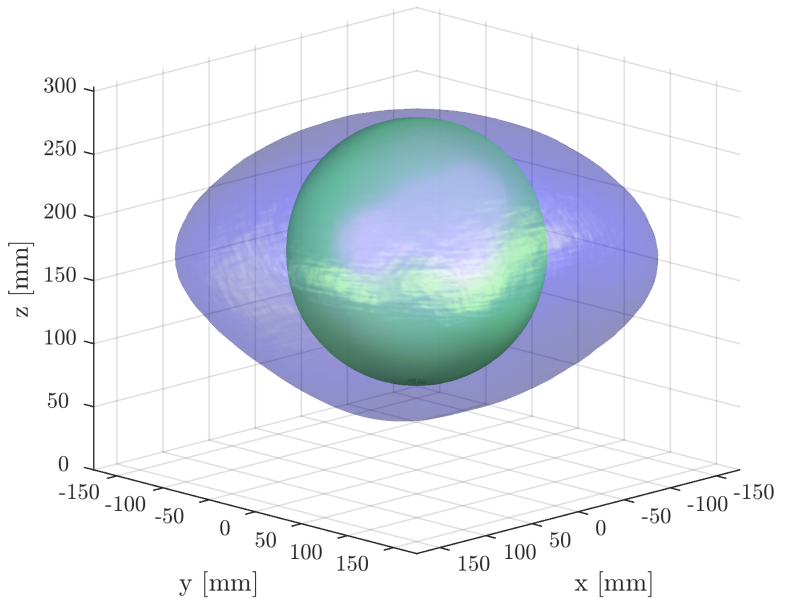

Fig. 6. Manipulation restriction based on dexterity measure, green: permitted workspace, blue: reachable workspace.

where the joint limit measure $\mathcal{L}_{q}$ are augmented onto the manipulability measure $\mathcal{M}$, and where $\mathcal{L}_{q}$ is defined as

$$
\mathcal{L}_{q}=1-\exp \left\{-\kappa \prod_{i=1}^{n_{k}} \frac{\left(q_{i}-q_{i, \min }\right)\left(q_{i, \max }-q_{i}\right)}{\left(q_{i, \max }-q_{i, \min }\right)^{2}}\right\} .
$$

Evaluating the dexterity of random tool configurations based on the end-effector position provides a map which depicts regions of low (blue) and high (red) dexterity, as shown in Fig. 5. Voxel entries in this map are generated by recording the maximum dexterity value obtained across multiple tool configurations for a given end-effector position. This further allows the definition of a dexterity-dependent threshold boundary, limiting the permitted manipulation workspace to areas of sufficient dexterity, see Fig. 6. Using simple geometric primitives such as spheres, capsules or convex hulls, the restricted workspace is enforced by projecting the user defined poses onto the permitted workspace to obtain the desired end-effector pose $\boldsymbol{T}_{e, d}$. To avoid slack in the manipulation, $\boldsymbol{T}_{e, d}$ follows the set-point if it is moved back towards the inside of the restricted workspace.

\section{Gaze-Assisted Adaptive Motion Scaling}

Motion scaling has always been considered a clear advantage of robotic systems, allowing the surgeon to perform much finer movements than would be possible by hand. However, the same scaling factor can make moving the instruments around the surgical scene time consuming and tedious by forcing the user to clutch and reposition the master device end-effectors. This behaviour is particularly noticeable on master devices with small workspaces, as users will often reach the edge of the workspace while performing larger movements. While it is possible to change the value of the scaling factor manually on some systems, this does not constitute a practical solution as the surgeon is effectively substituting clutching for manually toggling between different scaling factors. This paper proposes to use eye-tracking data in conjunction with multiple other sources of information available from the robotic system to infer where the surgeon is trying to reach. This information is then used to modulate the motion scaling factor such that distant targets are reached more quickly. In this paper, the authors consider that an increase in the scaling factor generate faster robot motions, and a decrease in the scaling factor generate slower robot motions.

\section{A. Definition of Aims and Input Data}

The proposed approach seeks to fulfil the following aims:

i To be safe, such that surgeons never encounter undesirable motions or unpredictable system behaviour,

ii To noticeably improve the speed at which distant goals can be reached by reducing the need for clutching,

iii To fulfil the above goals without sacrificing the capability to perform fine movements by taking full advantage of motion scaling,

iv To fulfil the above goals using an implicit approach, i.e. without introducing additional inputs for the user to handle.

The choice of motion scaling modulation displays some clear advantages to achieve these goals. In particular, adaptive motion scaling presents an inherent safety as any motion performed by the robot has to be directly generated by the user. However, in order to modulate the motion scaling in an effective manner the user's intention must be inferred by the system. The core premise behind the proposed approach stems from the observation that, when reaching for an object, humans fixate the object they are trying to reach and not their hands. Similar behaviour can be observed in a variety of disciplines, from sports to instruments: once an individual is proficient enough in a task, they tend to fixate their destination and not their tool. The same principle is exploited here to determine where the user is trying to move the surgical instruments. In order to elaborate on this concept, several different types of information are needed. Firstly, the system must be able identify where the user is looking with regards to the instruments. This necessitates the use of an eye-tracker, but also to track the instruments in the camera image. To further assess the user's intention, the hand motions performed using the master devices are also recorded. By combining gaze and tool tracking information with the direction in which the user is moving the master device end-effectors as well as its speed, the system is capable of further improving its evaluation of the user's intention. Lastly, the homogeneous transformations of the robotic arms are necessary to bring these different sources of information into the same reference frame. In summary the following input data is required:

- Eye-tracking data,

- Tool tracking information,

- Hand motions (from the master device),

- Homogeneous transformations of the robotic arms.

\section{B. Instrument Tracking}

A detection-and-tracking approach was implemented to find the 2D locations of the robotic tools. This approach 
consists of a colour-based detector that localises the endeffector of the tool (defined here as the wrist joint), which was coloured with a green dot. As the instrument shape can change significantly depending on its kinematic configuration and cause the detector to fail, a tracker was included to consider the temporal information of the tool motion. The salient image gradient features [16] were extracted inside a bounding box that encloses the end-effector. As can be seen from Fig. 7, these gradient features identify well the tool contours. We then perform forward-backward tracking based on optical flow [17] to estimate the locations of these gradient features in subsequent images. To determine the location of the tool in the current image, we consider the 2D offsets of the features and calculate the location and size of the bounding box. The location of the tool from the tracker is then defined as the centre of the box in the current image. Together the detector and temporal tracker provided a robust tool tracking method running at over 30 frames per second.

\section{Adaptive Scaling Generation}

The aim of the adaptive motion scaling is to generate a value $k_{a s}$ contained between 0 and 1 such that the final scaling factor of the system $k_{s t}$ is defined as:

$$
k_{s t}=s_{b} \times\left(1+s_{c} \times k_{a s}\right),
$$

where $s_{b}$ is base scaling factor of the system (a typical value for robotic systems being 0.25 for a ratio of 1 to 4 ) and $s_{c}$ is the scaling coefficient multiplier attributed to adaptive scaling. Using the input data detailed in section IV-A, three main contributing factors to $k_{a s}$ can be listed:

1) The distance between the gaze fixation point and the instrument detected in the image. Higher distances should result in a higher scaling value, while the scaling should not be increased when the user is looking close to the tool as this does not indicate a desire to reach a distant location.

2) The angle between the vector generated from the instrument tip to the fixation point and the hand motion. Lower angle values should result in a higher scaling value as the user would be moving the robot in the direction where he is looking. High angle values should not increase the scaling value.

3) The speed at which the user is moving the master device end-effectors. Low speeds could indicate that the user is not trying to reach a distant objective, and as such the scaling factor should not be increased.
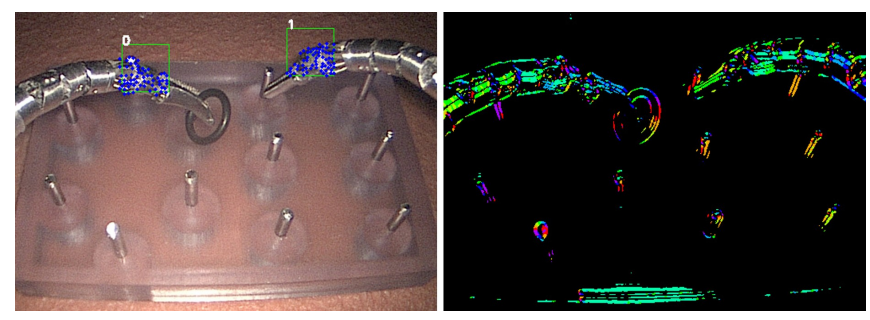

Fig. 7. Tool tracking. Left: temporal feature tracking and bounding boxes. Right: salient image gradients.

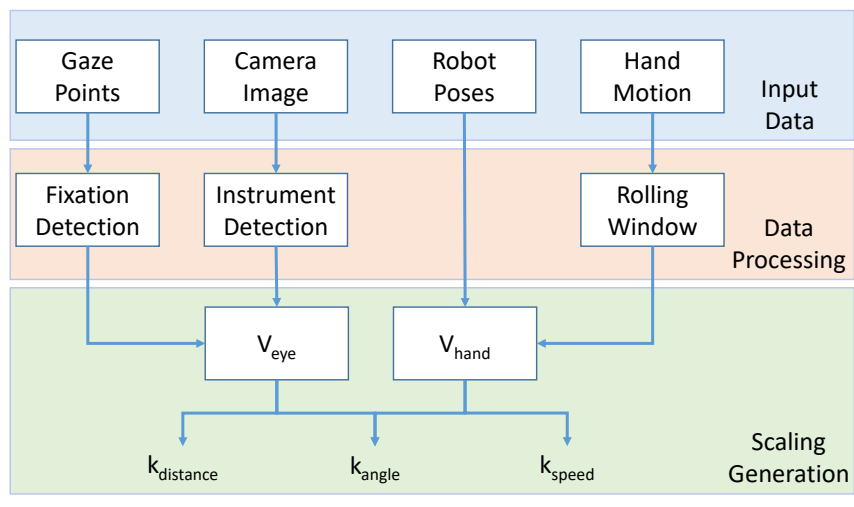

Fig. 8. Input data flow for the generation of the scaling factor.

These three factors are respectively written as $k_{d}, k_{a}$, and $k_{s}$, and can all range from 0 to 1 . The contribution of the adaptive scaling can then be defined as:

$$
k_{a s}=k_{d} \times k_{a} \times k_{s} .
$$

Fig. 8 displays the flow of information used to generate the each scaling factor. In order to define these three factors, the two following vectors must be defined:

$$
\begin{aligned}
V_{\text {eye }} & =P_{\text {fixation }}-P_{\text {tool }}, \\
V_{\text {hand }} & =P_{\text {hand }_{t}}-P_{\text {hand }_{t-t_{w}}},
\end{aligned}
$$

where $P_{f}$ is the user's 2D fixation point on the screen, $P_{\text {tool }}$ is the detected 2D position of the instrument on the screen, $P_{\text {hand }_{t}}$ is the position of the hand at the present time $t$ as obtained from the master device, and $P_{\text {hand }_{t-t}}$ is the position of the hand at a previous time based on a specific time window duration $t_{w}$. To obtain the fixation points from the eye tracking data, a Dispersion-Threshold Identification (ID-T) approach was used [18], [19]. Note that $V_{\text {hand }}$ is a 2dimensional vector obtained from the projection of the hand motion at specific time intervals $t_{w}$ onto the camera plane. Using these definitions, each of the three factors comprising $k_{a s}$ can be defined:

$$
\begin{aligned}
k_{d} & =\frac{\min \left(\left\|V_{\text {eye }}\right\|, d_{\mathrm{pmax}}\right)}{d_{\mathrm{pmax}}}, \\
k_{a} & =\frac{\min \left(\max \left(0, a_{c}-a_{\min }\right), a_{\max }-a_{\min }\right)}{a_{\max }-a_{\min }}, \\
k_{s} & =\frac{\min \left(\left\|V_{\text {hand }}\right\| / t_{w}, s p_{e}\right)}{s p_{e}},
\end{aligned}
$$

where $d_{\text {pmax }}$ is the distance in pixels under which $k_{d}$ starts to decrease linearly, $a_{c}$ is the angle between $V_{\text {eye }}$ and $V_{\text {hand }}, a_{\text {min }}$ is the angle below which $k_{a}$ is maximum (value of 1), $a_{\max }$ the angle above which $k_{a}$ is minimum (value of 0 ), and $s p_{e}$ is the speed under which $k_{s}$ starts to decrease linearly. The different parameters presented were fine-tuned over multiple iterations of the system to provide the responsiveness desired. Table I displays the values used for the user study. 
TABLE I

ADAPTIVE SCALING PARAMETERS.

\begin{tabular}{|c|c|c|c|c|}
\hline$s_{c}$ & $t_{w}(\mathrm{~s})$ & $a_{\min }(\mathrm{deg})$ & $a_{\max }(\mathrm{deg})$ & $s p_{e}(\mathrm{~mm} / \mathrm{s})$ \\
\hline 1.3 & 0.1 & 20 & 40 & 20 \\
\hline
\end{tabular}

To avoid sharp changes in the scaling factor due to single steps in the algorithm, a moving window spanning 100 milliseconds (same value as $t_{w}$ and the fixation detection window from the ID-T algorithm) was introduced to smooth the results out. As eye tracking and tool detection can both fail for various reasons (e.g. tools missing from the image, user looking away), an additional layer of safety was introduced in the implementation of the adaptive scaling algorithm. In case either the instrument or the user's gaze fixation point is not found, both $k_{d}$ and $k_{a}$ are considered invalid and set to zero.

\section{Behavioural analysis}

Fig. 9 shows a typical motion benefiting from the adaptive motion scaling. In the first part of the figure (top left image, graphs highlighted in light red) the user has just started looking at the new destination for the left instrument and the motion has not even started. As such the ID-T algorithm has not had time to register a fixation and so both $k_{d}$ and $k_{a}$ have a value of 0 . By the time the fixation is registered the motion of the master device in the correct direction has already begun, and so the scaling factor $k_{a s}$ increases as all conditions are met. In the second phase the scaling factor reaches its maximum value as the motion carries on in the right direction. Finally, upon reaching the desired location $k_{d}$ drops significantly, followed by the other coefficients as the user is no longer fixating on the same point.

\section{EXPERIMENT}

\section{A. Experimental Setup}

A user study was conducted to evaluate the usability and effectiveness of the adaptive motion scaling. The chosen task was designed to require large motions from the robotic instruments, while still requiring the precision of a normal scaling factor. Users were asked to pass a rubber ring between the two instruments, by moving over a large peg board. The rubber ring started in the gripper of the right instrument, with both instruments on opposing side of the bottom of a peg board. The left instrument would come grab the ring from the right tool and bring it back on the left side. The right instrument would then repeat the same procedure, finishing the manoeuvre on the right-hand side of the row above. The task carried on in this manner until the top of the board was reached, for a total of nine tool manoeuvres. Nine non-clinical novices were presented to the system and given time to familiarize themselves with the controls. They were then trained on the task twice, once with adaptive motion scaling and once without. After training, each participant would conduct the experiment once with adaptive motion scaling and once without in a randomized manner.
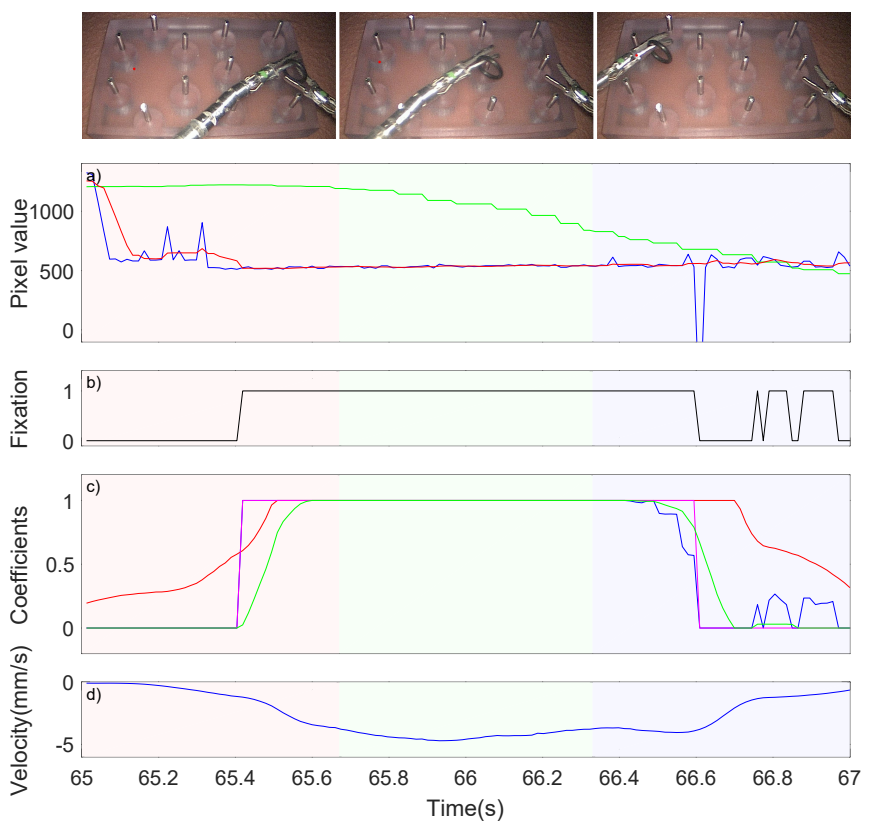

Fig. 9. Evolution of scaling coefficients from input data. Top: from left to right, images of a ring transfer from the right instrument to the left instrument in chronological order. The red dot represents the gaze point. a) Raw X-axis gaze data pixel values (blue), gaze fixation X-axis data pixel values (red), and left instrument end-effector $\mathrm{X}$-axis pixel values (green). b) validity of fixation data (fixation identified: value of 1,0 otherwise), c) $k_{d}$ (blue), $k_{a}$ (magenta), $k_{s}$ (red), and $k_{a s}$ (green). d) $V_{h a n d} \mathrm{X}$-axis value in $\mathrm{mm} / \mathrm{s}$ (blue)

\section{B. Results}

The results of the study are presented in Fig. 10. As can be seen, the use of the adaptive scaling provided a highly significant improvement in both task execution times and in the number of times the system had to be clutched. User feedback was strongly in favour of the system, which can be explained by the reduction in frustration at having to constantly clutch and reposition the master manipulator. This is likely emphasized by the fact that the Omega.7 master devices used have a small workspace. Fig. 11 further illustrates this point through heatmaps of the eyetracking data
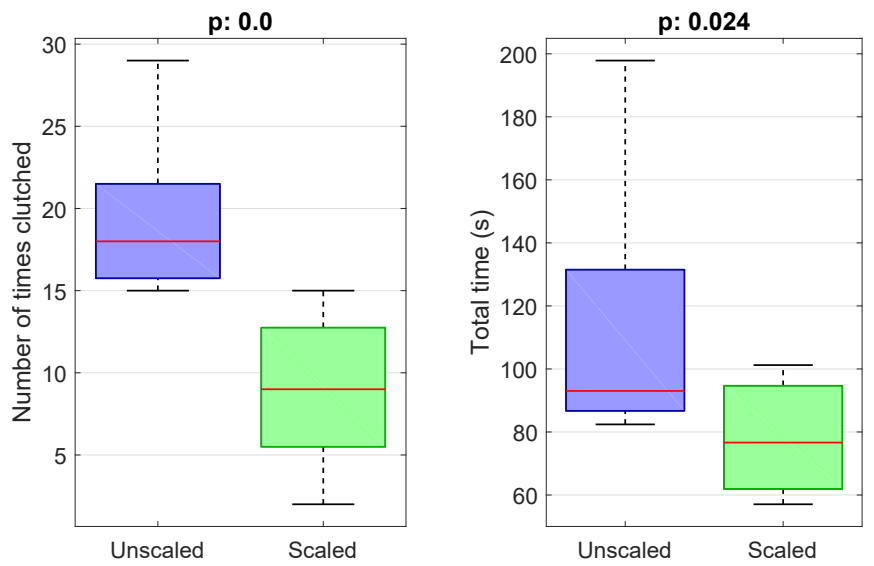

Fig. 10. User study comparing task execution times and number of times the system was clutched across nine participants. 

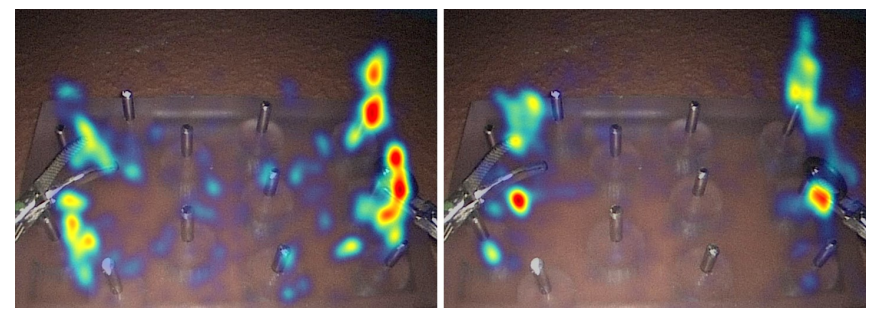

Fig. 11. Eye-tracking heatmaps. Left: without adaptive scaling. Right: with adaptive scaling.

obtained from both tasks. The lack of adaptive scaling is clearly noticeable by the user having to constantly look back at the instruments to clutch the master devices.

\section{CONCLUSION}

This paper presents implicit, gaze-assisted adaptive motion scaling for highly articulated instrument manipulation. It formalizes the differential inverse kinematic approach used to control a redundant 9-DoF virtual tool, suitable to operate in tight spaces with little or no triangulation and close to jointlimits, while also providing highly dexterous manipulation in conventional laparoscopic procedures. Furthermore, a gazeassisted control scheme was presented using implicit operator input to seamlessly scale-up master-device motions and allow more effective manipulation. The developed system infers the user's intention and facilitates time-efficient motions, as well as reduces the need for clutching in an inherently safe manner. Further work will focus on developing more advanced tool tracking methods to recover the entire kinematic pose of the instruments, as well as expanding the data fusion techniques presented in this work to improve other aspects of robotic control such as camera control.

\section{REFERENCES}

[1] J. Burgner-Kahrs, D. C. Rucker, and H. Choset, "Continuum robots for medical applications: A survey," IEEE Transactions on Robotics, vol. 31, no. 6, pp. 1261-1280, Dec 2015.

[2] V. Vitiello, S. L. Lee, T. P. Cundy, and G.-Z. Yang, "Emerging robotic platforms for minimally invasive surgery," IEEE Reviews in Biomedical Engineering, vol. 6, pp. 111-126, 2013.

[3] A. Bajo, R. E. Goldman, L. Wang, D. Fowler, and N. Simaan, "Integration and preliminary evaluation of an insertable robotic effectors platform for single port access surgery," in IEEE International Conference on Robotics and Automation (ICRA), May 2012, pp. 33813387.

[4] H. J. Marcus, A. Hughes-Hallett, T. P. Cundy, G.-Z. Yang, A. Darzi, and D. Nandi, "da vinci robot-assisted keyhole neurosurgery: a cadaver study on feasibility and safety," Neurosurgical Review, vol. 38, no. 2, pp. 367-371, 2015.
[5] H. Han, J. Lee, and J. Park, "A continuous task transition algorithm for operational space control framework," in 9th International Conference on Ubiquitous Robots and Ambient Intelligence (URAI), Nov 2012, pp. $148-152$.

[6] F. Chaumette and E. Marchand, "A redundancy-based iterative approach for avoiding joint limits: application to visual servoing," IEEE Transactions on Robotics and Automation, vol. 17, no. 5, pp. 719-730, Oct 2001.

[7] A. Kapoor, N. Simaan, and R. Taylor, "Suturing in Confined Spaces: Constrained Motion Control of a Hybrid 8-DOF Robot," International Conference on Advanced Robotics, pp. 452-459, 2005.

[8] J. Lee, J. Kim, K. K. Lee, S. Hyung, Y. J. Kim, W. Kwon, K. Roh, and J. Y. Choi, "Modeling and control of robotic surgical platform for single-port access surgery," IEEE International Conference on Intelligent Robots and Systems (IROS), pp. 3489-3495, 2014.

[9] K. Leibrandt, P. Wisanuvej, G. Gras, J. Shang, C. A. Seneci, P. Giataganas, V. Vitiello, A. Darzi, and G.-Z. Yang, "Effective Manipulation in Confined Spaces of Highly Articulated Robotic Instruments for Single Access Surgery," IEEE Robotics and Automation Letters (RAL), vol. 3766, no. c, pp. 1-8, 2017.

[10] D. P. Noonan, G. P. Mylonas, J. Shang, C. J. Payne, A. Darzi, and G.-Z. Yang, "Gaze contingent control for an articulated mechatronic laparoscope," in 3rd IEEE RAS and EMBS International Conference on Biomedical Robotics and Biomechatronics (BioRob), Sept 2010, pp. 759-764.

[11] K. Fujii, A. Salerno, K. Sriskandarajah, K.-W. Kwok, K. Shetty, and G.-Z. Yang, "Gaze contingent cartesian control of a robotic arm for laparoscopic surgery," in IEEE/RSJ International Conference on Intelligent Robots and Systems, Nov 2013, pp. 3582-3589.

[12] G. Gras and G.-Z. Yang, "Intention recognition for gaze controlled robotic minimally invasive laser ablation," in IEEE/RSJ International Conference on Intelligent Robots and Systems, 2016, pp. 2431-2437.

[13] I. Tong, O. Mohareri, S. Tatasurya, C. Hennessey, and S. Salcudean, "A retrofit eye gaze tracker for the da vinci and its integration in task execution using the da vinci research kit," in Intelligent Robots and Systems (IROS), 2015 IEEE/RSJ International Conference on. IEEE, 2015, pp. 2043-2050.

[14] C. A. Seneci, J. Shang, A. Darzi, and G.-Z. Yang, "Rapid manufacturing with selective laser melting for robotic surgical tools: Design and process considerations," in IEEE/RSJ International Conference on Intelligent Robots and Systems (IROS), Sept 2015, pp. 824-830.

[15] M. J. Tsai and Y. H. Chiou, "Manipulability of manipulators," Mechanism and Machine Theory, vol. 25, no. 5, pp. 575 - 585, 1990.

[16] M. Ye, L. Zhang, S. Giannarou, and G.-Z. Yang, "Real-time 3d tracking of articulated tools for robotic surgery," in International Conference on Medical Image Computing and Computer-Assisted Intervention. Springer, 2016, pp. 386-394.

[17] Z. Kalal, K. Mikolajczyk, and J. Matas, "Forward-backward error: Automatic detection of tracking failures," in Pattern Recognition (ICPR), 2010 20th International Conference on, Aug 2010, pp. 27562759.

[18] D. D. Salvucci and J. H. Goldberg, "Identifying fixations and saccades in eye-tracking protocols," in Proceedings of the 2000 symposium on Eye tracking research \& applications. ACM, 2000, pp. 71-78.

[19] P. Blignaut, "Fixation identification: The optimum threshold for a dispersion algorithm," Attention, Perception, \& Psychophysics, vol. 71, no. 4, pp. 881-895, 2009. 